\title{
SIMULATION OF CROWD EVACUATION BEHAVIOUR IN OUTDOOR PUBLIC PLACES - A MODEL BASED ON SHANGHAI STAMPEDE
}

\author{
Liu, S. S. ; Liu, J. ${ }^{* * \#} \&$ Wei, W.** \\ ${ }^{*}$ School of Management, Hubei University of Education, Wuhan, P. R. China \\ ** School of Information and Safety Engineering, Zhongnan University of Economics and Law, \\ Wuhan, P. R. China \\ *** College of Business, Arkansas Tech University, Russellville, USA \\ E-Mail: whutrobin@ 163.com ( ${ }^{\#}$ Corresponding author)
}

\begin{abstract}
The crowd motion in complex sites is a critical basis of analysing the law of crowd evacuation behaviour. However, few studies comprehensively considered the overall effect of pedestrians and the microscopic features of individuals. To find out the behavioural law of pedestrians in outdoor public places during evacuation, the simulation system proposed in this study utilized the measured geographic data of the Shanghai Bund where a serious stampede accident occurred on December 31, 2014. The behavioural parameters were first established including the pedestrian's shoulder widths and walking speeds. Afterwards, the crowd motion in Shanghai Bund was simulated based on Pathfinder platform. Results show as the following: (1) there exist "walking-along-the-sides effect", "imitation effect" and "non-optimal effect" in the evacuation process; (2) the time of crowd evacuation varies according to physiological characteristics, movement trajectories, and destinations. These conclusions may provide recommendations for crowd evacuation in public places.

(Received in September 2018, accepted in January 2019. This paper was with the authors 1 month for 2 revisions.)
\end{abstract}

Key Words: Outdoor Public Places, Crowd Evacuation Effects, Pathfinder, Shanghai Stampede

\section{INTRODUCTION}

According to incomplete statistics, 41 crowd stampedes worldwide have resulted in at least 2683 deaths and 3014 injuries since 2007, and more than half of these stampedes occurred in outdoor public places. Characterized by openness, publicity and complexity, outdoor public places can easily attract large crowds. If these crowds cannot evacuate properly, then crowd chaos, and even stampede, are likely to occur. Crowd stampede means crowded people push one another disorderly because of nervous tension, fear, or disturbance during evacuation, thereby resulting in trampling of people and casualties [1]. Most stampede accidents occurred when crowds failed to effectively evacuate [2]. Therefore, analysing the evacuation behaviours and summarizing the law of crowd evacuation are the key to prevent crowd stampede.

Current studies mainly focus on macroscopic [3, 4] and microscopic models [5] to analyse crowd evacuation. Macroscopic models regarded crowd movement as the movement of gas [6] or liquid [7] and explored the movement law of the crowd from an overall perspective but neglected the diverse characteristics of individuals [8]. Microscopic models focused on the microscopic features of individuals [9] by considering their intentions, desired speeds, and interactions among individuals [10]. However, studies that integrate the diversity of individual pedestrians with the evacuation effects of large-scale crowd are limited. Moreover, crowd movement consists of individual pedestrians with diversified physiological and behavioural characteristics. Group effect of large-scale crowd evacuation is an important law of crowd movement. Simulating the crowd movement in that situation can be helpful in exploring the principle of crowd evacuation and in guiding the crowd to properly disperse 
during emergencies. Therefore, it is of great important to find out the principle of crowd evacuation with the combination of pedestrians' diversity, flow trajectories, and the real characteristics of outdoor public places.

This study developed a crowd evacuation model by using Pathfinder. The 2014 Shanghai Stampede was used as the background. The simulation and behaviour parameters were set according to the physiological data of 350 tourists at the Bund who were randomly selected to model the effects of crowd evacuation. Relevant study conclusions may provide recommendations of the management and control of crowd evacuation.

\section{STATE OF THE ART}

Studies on crowd evacuation can be classified into macroscopic and microscopic models. Research on macroscopic models performed analogies among pedestrian flow and fluid [11] or other substances. Henderson [11] depicted an analogy between pedestrian flow and fluid and proposed the gas dynamic equation of crowd evacuation by using the MaxwellBoltzmann distribution in thermodynamics. However, this method is not applicable to large population densities [7]. Hughes [7] examined crowd behaviours according to the theory of media, made an analogy between crowd movement and fluid movement to construct the fluiddynamic model, and analysed and demonstrated the law of crowd movement. Di Francesco et al. [4] and Amadori and Di Francesco [12] demonstrated the law of crowd movement from microscopic perspective in further through calculation and simulation based on Hughes' model. However, the Hughes model has limitations in interrupted crowd movement and congestion in narrow space. In Twarogowska' s paper, the Hughes model was improved and optimized by using the Eikonal equation [3]. To examine pedestrian movement, Twarogowska et al. [3] constructed Hughes and second-order differential equation model by using Eikonal equation. The results showed that the second-order differential equation model solved the problem of congestion in narrow areas. However, the relationship between diverse pedestrians in the aspect of dynamic analysis of complex congestion was difficult to explain $[3,13]$.

Most existing studies are based on microscopic models, such as social force [9, 12, 14, 15], lattice gas [16, 17], cellular automata [13, 18], and agent [19-22] models. (1) Social force model. Helbing and Molnar [14] established the social force model considering pedestrian motion, which was affected by the sum of attractive, repulsive, driving, and fluctuating forces. The practical importance of the social force model was also verified in the simulations. Zeng et al. [9] investigated the effect of restrictive pedestrian behaviours on crowd evacuation by using social force model, such as the rule of avoiding conflict and responding to signal lights. Gao et al. [15] optimized the social force model by considering the competition among pedestrians. However, these studies neglected the influence of heterogeneities, such as age, gender, and teamwork of different pedestrians, on crowd movement [9]. (2) Lattice gas model. Helbing et al. [16] simulated pedestrian flows based on the data acquired from video experiment and the lattice gas model to comparatively analyse the law of crowd evacuation. Guo et al. [17] constructed a heterogeneous lattice gas model to investigate the evacuation of pedestrians during fire. Results showed that high temperature affected pedestrian's path choice and cause congestion. (3) Cellular automata model, which is widely used in crowd evacuation research, is a kind of discrete dynamic model. Li and Han [13] constructed the cellular automata model based on high familiarity and aggressiveness behaviours to study their effect on crowd evacuation. Similarly, Huang et al. [18] constructed a cellular automata model to discuss the influence of environmental characteristics and friendly behaviours on emergency evacuation and demonstrated self-organization phenomena in crowd evacuation. These scholars discussed the influence of pedestrian behaviours on crowd evacuation by using the cellular automata model. However, only several behaviours were considered. In addition, 
the simulation form was not intuitive or stereoscopic. (4) Agent model. The parameters in the agent model can be set flexibly according to different situations of various people so that the intelligent agent can have several pedestrian capacities, such as autonomous, social, reaction, learning, and cooperation abilities [20]. Joo et al. [20] constructed finite state automata model (FSA) based on the ecological concept of affordance theory and the agent model to explore pedestrian's behaviours under emergency evacuation. Tan et al. [21] assumed that the evacuees were familiar with the internal structure of the building and investigated the evacuation characteristics of pedestrians in the building by using the multi-agent model. Was and Lubas [22] combined the multi-agent model and the cellular automata model to analyse the congestion dynamics of pedestrians. The agent model is widely used in the study of crowd evacuation because its intelligence is similar to human characteristics. However, most existing studies focused on individual characteristics, and few of them combined the whole crowd evacuation effects with individual characteristics.

Overall, among the current studies on crowd evacuation, macroscopic models mainly focus on revealing the overall law of crowd movement but ignore the complex characteristics of individuals. Microscopic models focus on individual characteristics but pay less attention to the overall effects of crowd evacuation. Few literatures combine the diverse physiological characteristics of pedestrians with their actions strategies to reveal the overall effects of crowd evacuation. Therefore, the simulation scenarios were set by measuring the terrain data of the place by using Chen Yi Square on the Bund of Shanghai in China as the background. Moreover, the pedestrian parameters were set according to the typical body characteristics of pedestrians to construct a $3 \mathrm{D}$ dynamic crowd evacuation simulation model, so as to simulate the crowd evacuation law in this place.

The rest of this study is organized as follows: Section 3 constructs the simulation model of crowd evacuation in outdoor public places based on the collected data and simulation hypothesis. Section 4 implements several simulations to explore the effects of crowd evacuation, to validate the model, and to condense the characteristics of crowd evacuation. Finally, Section 5 provides conclusions and further work.

\section{METHODOLOGY}

\subsection{Formulation of simulation model}

The formulation of the simulation model originated from the place where Shanghai Stampede occurred. At 11:35 p.m. on December 31, 2014, a large number of tourists and citizens gathered at the Bund of Shanghai celebrating the New Year. Some people fell down the stairs at the pedestrian passageway, which connects the south-eastern corner of Chen Yi Square with the Huangpu River viewing platform. Then, numerous pedestrians fell and overlapped, thereby causing stampede which resulted in 36 deaths and 49 injuries. The simulation model was constructed by using Pathfinder software to explore the law of crowd evacuation in outdoor public places based on the measurement of the place where the event occurred.

Pathfinder software is a human movement simulator based of agent model, which can provide 3D visualization results. The simulation of Pathfinder software can be classified into two modes: SFPE and steering. The walking speed of pedestrians in the SFPE mode is determined by the room crowd density. Pedestrians crowd around one another rather than avoiding pushing others. The movements of pedestrians in steering mode are controlled by path planning, guidance mechanism, and collision processing. The algorithm generates a new path when the distance between pedestrians and the nearest point exceeds a certain threshold, thereby changing the pedestrian trajectory [23]. The steering model can remarkably reflect the actual law of crowd evacuation. Thus, it was applied to this study. The calculation of 
pedestrian evacuation movement usually includes the following steps based on the basic principles of Pathfinder [24]:

(1) The initial position velocity and acceleration are given by equation:

$$
V_{0}=\left\{\begin{array}{cc}
V_{\text {max }} \frac{k}{1.4} & \left(D<0.55 \text { pers } / \mathrm{m}^{2}\right) \\
V_{\text {max }} \frac{k-0.266 k D}{1.19} & \left(D \geq 0.55 \text { pers } / \mathrm{m}^{2}\right)
\end{array}\right.
$$

where $V_{0}(\mathrm{~m} / \mathrm{s})$ is the velocity of people in the initial position. $V_{\max }(\mathrm{m} / \mathrm{s})$ is the maximum velocity of people. $k$ is the influencing factor of evacuation velocity, and its value varies according to the slope, so take 1.4 in plane 1 when the stairs are 7.5 feet high, and 1.23 when the steps are 1.23 feet high. $D\left(\right.$ pers $\left./ \mathrm{m}^{2}\right)$ is the population density.

$$
a_{\max }=\frac{V_{\max }}{t_{\text {accel }}}
$$

where $a_{\max }\left(\mathrm{m} / \mathrm{s}^{2}\right)$ is the maximum acceleration, $t_{\text {accel }}(s)$ is the acceleration time.

(2) The evacuation direction is determined by the following formula:

$$
C_{\text {seek }}=\frac{\theta_{t}}{2 \pi}
$$

where $C_{\text {seek }}$ is the evacuation cost in a certain direction, and $\theta_{t}$ is the angle between the velocity direction and the optimal evacuation curve tangent.

(3) The velocity in the evacuation direction is determined by the following formula:

$$
\begin{gathered}
D_{\text {stop }}=\frac{v_{\text {curr }}^{2^{\prime}}}{2 a_{\max }} \\
\left|\overline{v_{\text {des }}}\right|=\left\{\begin{array}{cl}
0 & D_{\max } \leq D_{\text {stop }} \\
v_{\max } & D_{\text {max }}>D_{\text {stop }}
\end{array}\right. \\
\overline{v_{\text {des }}}=\left|\overline{v_{\text {des }}}\right| \overline{d_{\text {des }}}
\end{gathered}
$$

where $D_{\max }$ is the maximum distance for the lowest cost sample direction, $\overline{d_{d e s}}$ is the lowest cost sample direction, $v_{\text {curr }}$ is the occupant's current velocity, and $\overline{v_{d e s}}$ is the desired velocity.

(4) Acceleration is calculated as:

$$
\bar{a}=\frac{\overline{v_{\text {des }}}-\overline{v_{\text {curr }}}}{\left|\overline{v_{\text {des }}}-\overline{v_{\text {curr }}}\right|} a_{\max }
$$

(5) The velocity and position vector at the evacuation arrival place can be formulated as follows:

$$
\begin{gathered}
\overline{v_{\text {next }}}=\overline{v_{\text {curr }}}+\bar{a} \Delta t \\
\overline{p_{\text {next }}}=\overline{p_{\text {curr }}}+\overline{v_{\text {next }}} \Delta t
\end{gathered}
$$

where $\Delta t$ is the time step, $\overline{p_{\text {curr }}}$ is the present position, and $\overline{p_{\text {next }}}$ is the next position at the next unit time.

\subsection{Simulation hypothesis}

The following hypotheses were made to investigate the evacuation law on the Bund of Shanghai. 
In order to simplify the model, situation that the pedestrians entered in the study area at any time was not taken into account. In other words, it is assumed that pedestrians in the area only exited.

Pedestrians were assumed to have no preference for any exit in the Bund and know how to escape the area from an exit in advance.

\subsection{Scenario construction}

The Bund is a public area in Huangpu District, which covers an area of 3.1 square kilometers, from Huangpu River flood wall in the east to the west pavement of East Zhongshan No. 1 and No. 2 Road in the west, from the north pavement of Dongmen Road in the south to the north Bank of Suzhou Rive in the north. Chen Yi Square is located in the middle of the Bund (facing the road between No. 335 to No. 309 of East Zhongshan No. 1 Road), which is adjacent to the east end of Nanjing East Road and connected with East Zhongshan No. 1 Road. This place covers a public area of approximately 2877 square meters. Huangpu River viewing platform, which is connected with Chen Yi Square via huge steps and ramps, is the best viewing position on the Bund. In addition, the transportation around Chen Yi Square is convenient. It is approximately 580 meters away from Rail Transit Line 2 and East Nanjing Road Line 10. Therefore, it is the area with the largest pedestrian flows in the Bund. Visitors can watch the landscape along both banks of the Huangpu River by stepping up to the viewing platform because it is the highest point of the Bund.

The research scope of this study is the public area that extends 145 meters northward and 108.5 meters southward and takes the viewing platform as the centre. The area consists of three floors. The first floor is a public activity area where main buildings, such as Chen Yi Square and Bund Commercial Bodies, are located. The second floor is the viewing platform layer (low). The third floor is the viewing platform (high). According to field measurement, the 3D effect map of the study area was constructed in Pathfinder.

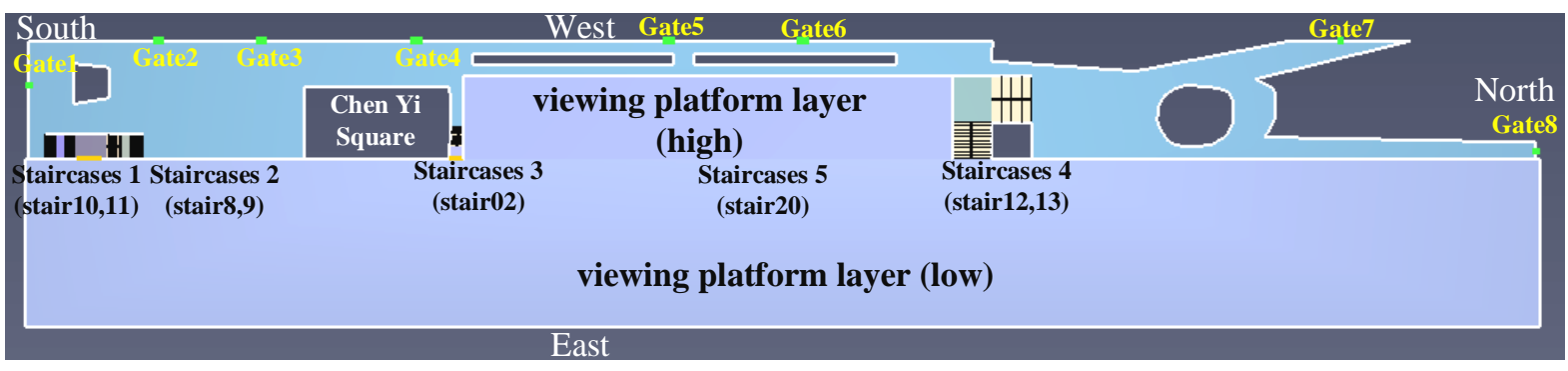

Figure 1: 3D effect aerial view of the study area.

\subsection{Parameter setting}

The parameters of gates, the locations of staircases, and obstacles were set based on measurement. Pedestrians were randomly selected to measure their shoulder widths and walking velocities so as to set the parameters of pedestrians. The details are presented as follows.

(1) Setting of gates

The evacuation routes of pedestrians on the Bund of Shanghai went in and out mainly through two zebra crossings near Chen Yi Square and the bus stop in the northwest. Therefore, the gates were set in the following places: Gate 1 at the south of Chen Yi Square; Gate 2 and Gate 3 at the area that connect two zebra crossings on East Zhongshan No.1 Road on the Southwest of Chen Yi Square; Gate 4 was set between Gate 2 and 3, where a large number of people went in and out; Gate 5 at the bus stop outside the commercial store under the viewing platform (high) where a large number of people went in and out; Gate 6 in the north of Gate 5, 
connecting the zebra crossing where a large number of people flow in and out; Gate 7 in the northwest corner of the commercial store where a road was located between the flower beds; Gate 8 in the northeast of the commercial store where a road was located between the flower beds. The width of Gate 7 and 8 was $1.5 \mathrm{~m}$, and other doors were $3 \mathrm{~m}$ wide.

(2) Setting of staircases

The staircases in the scenario were the same as the actual situation. Staircases 1 (stair 10, 11) and Staircases 2 (stair 8,9) were located in the southeast corner of Chen Yi Square, which connected the stair between Chen Yi Square and the viewing platform (low) $6 \mathrm{~m}$ wide. Staircase 3 (stair 02) was located in the north of Chen Yi Square, which connected the stair between Chen Yi Square and the viewing platform (low) $4.2 \mathrm{~m}$ wide. Staircase 4 (stair 12, 13) was located near the northern part of the commercial complex, which connected the stair between Chen Yi Square and the viewing platform (low), $9.6 \mathrm{~m}$ wide and was divided into the upper and lower parts. Staircase 5 (stair 20) connected the viewing platform (high) and the viewing platform (low), $120 \mathrm{~m}$ wide.

(3) Setting of obstacles

In Pathfinder, places where pedestrians were not allowed to enter were set as obstacles. According to the requirement, the following areas were set as obstacles: Chen Yi Square, flower beds in the west of the commercial complex, round flower beds, and two irregular flower beds in the north of the commercial complex.

(4) Setting of the pedestrian composition

Age and sex have great impact on crowd evacuation [25]. A total of 350 pedestrians on the Bund of Shanghai were randomly selected and divided into three groups, including 150 males, 150 females, and 50 children. The shoulder widths and walking velocities of these pedestrians were measured, and the maximums, minimums, means, and variances of these three groups of pedestrians were calculated (Table I). The shoulder widths and walking velocities of pedestrians on the Bund were assumed to be accorded with normal distribution.

Table I: Setting of typical pedestrians.

\begin{tabular}{|c|l|c|c|c|}
\hline \multicolumn{1}{|c|}{ Item } & \multicolumn{1}{|c|}{ Value } & Man & Woman & Child \\
\hline Shoulder width & Minimum value $(\mathrm{cm})$ & 39 & 34 & 21 \\
\cline { 2 - 5 } & Maximum value $(\mathrm{cm})$ & 51 & 42 & 33 \\
\cline { 2 - 5 } & Mean value $(\mathrm{cm})$ & 45.62 & 37.56 & 26.67 \\
\cline { 2 - 5 } & Variance & 2.63 & 1.45 & 3.43 \\
\hline \multirow{3}{*}{ Walking speed } & Minimum value $(\mathrm{m} / \mathrm{s})$ & 0.52 & 0.4 & 0.4 \\
\cline { 2 - 5 } & Maximum value $(\mathrm{m} / \mathrm{s})$ & 1.97 & 1.67 & 1.3 \\
\cline { 2 - 5 } & Mean value $(\mathrm{m} / \mathrm{s})$ & 1.31 & 1.13 & 0.82 \\
\cline { 2 - 5 } & Variance & 0.20 & 0.18 & 0.26 \\
\hline
\end{tabular}

The simulated pedestrian structure was set up according to the monitoring and statistics of daily pedestrian flow on the Bund of Shanghai and based on the research purpose shown in Table II.

Table II: Pedestrians structure on different floors.

\begin{tabular}{|c|l|c|c|c|c|}
\hline Floor & \multicolumn{1}{|c|}{ Description } & Total number & Man & Woman & Child \\
\hline Floor 1 & Chen Yi Square & 2000 & $45 \%$ & $45 \%$ & $10 \%$ \\
\hline Floor 2 & Viewing Platform (low) & 5000 & $45 \%$ & $50 \%$ & $5 \%$ \\
\hline Floor 3 & Viewing Platform (high) & 1000 & $45 \%$ & $45 \%$ & $10 \%$ \\
\hline
\end{tabular}




\section{RESULT ANALYSIS AND DISCUSSION}

\subsection{Simulation behaviour setting}

The complexity and variability of pedestrian evacuation behaviours, as well as the lack of relevant empirical data and observations [10], caused the following behaviours to be set through long-term observation at the simulation places and were based on the simulation purpose: Behaviour 1, going out from any exit; Behaviour 2, going to the viewing platform (high) first and then going out from any exit; Behaviour 3, waiting for $30 \mathrm{~s}$ and then going out from any exit; and Behaviour 4, waiting for $60 \mathrm{~s}$ and then going out from any exit. Various trajectories were set for pedestrians on different floors. The distributions of various behaviour on different floors are shown in Table III. The setting reflects the general state of tourists on the Bund, that is, some people have finished sightseeing and leave from any exit; some people go straight to the viewing platform to see the scenery, and then go out from any exits; and some people wait for a while and then take action.

Table III: Behaviour distributions of pedestrians on different floors.

\begin{tabular}{|c|c|c|c|c|c|}
\hline Floor & Total number & Behaviour 1 & Behaviour 2 & Behaviour 3 & Behaviour 4 \\
\hline Floor 1 & 2000 & $10 \%$ & $60 \%$ & $10 \%$ & $20 \%$ \\
\hline Floor 2 & 5000 & $30 \%$ & $40 \%$ & $20 \%$ & $10 \%$ \\
\hline Floor 3 & 1000 & $50 \%$ & $0 \%$ & $30 \%$ & $20 \%$ \\
\hline
\end{tabular}

\subsection{Summary of simulation results}

Simulations are implemented on the basis of the characteristic of pedestrian diversification, which shows that the evacuation simulation takes $16268.8 \mathrm{~s}$ in total, and the fastest evacuation takes $1.4 \mathrm{~s}$ while the slowest evacuation takes $16268.8 \mathrm{~s}$. The average evacuation time is $6837.2 \mathrm{~s}$ with the standard deviation of 4598.2. The shortest evacuation distance is $1.2 \mathrm{~m}$, and the longest evacuation distance is $3624.0 \mathrm{~m}$. Thus, the average evacuation distance is $539.2 \mathrm{~m}$, with the standard deviation of 499.3. Individual differences are observed in the crow evacuation simulation. The places, movement trajectories, and physiological characteristics of individuals all influence the evacuation time.

The speed during evacuation is very fast in the initial $100 \mathrm{~s}$. In this period, approximately 800 people are evacuated. Within 100 to $10000 \mathrm{~s}$, the number of evacuees increases steadily and tends to be stable. Within 10000 to $14000 \mathrm{~s}$, the evacuation speed accelerates, and the number of evacuees increases rapidly. After $14000 \mathrm{~s}$, the evacuation speed tends to be gentle. Fig. 2 illustrates that the crowd evacuation experiences four stages, namely, rapid, gentle, rapid, and gentle evacuation.

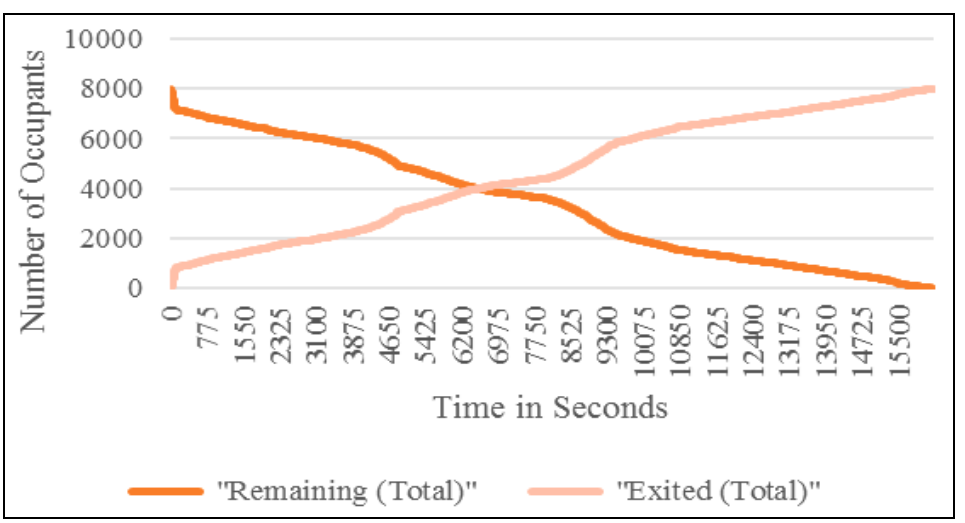

Figure 2: Change in the number of evacuees. 
The density curve (Fig. 3) for Staircase 3 (stair 12) shows that the crowd density is very high with the average of 450 people within 10519 s of evacuation. After $12577 \mathrm{~s}$, almost no pedestrians pass here. For Staircase 1 (stair 13), the pedestrian flows first increase and then reduce within 9929 s, and reach the maximum of 279 at 9467 s. After 9929 s, the pedestrian flows drop sharply. At Staircase 2 (stair 02), the pedestrian flows are approximately 100. Especially after 12,623 s, almost all the pedestrians gather at Staircase 2, and almost no pedestrian pass through other staircases.

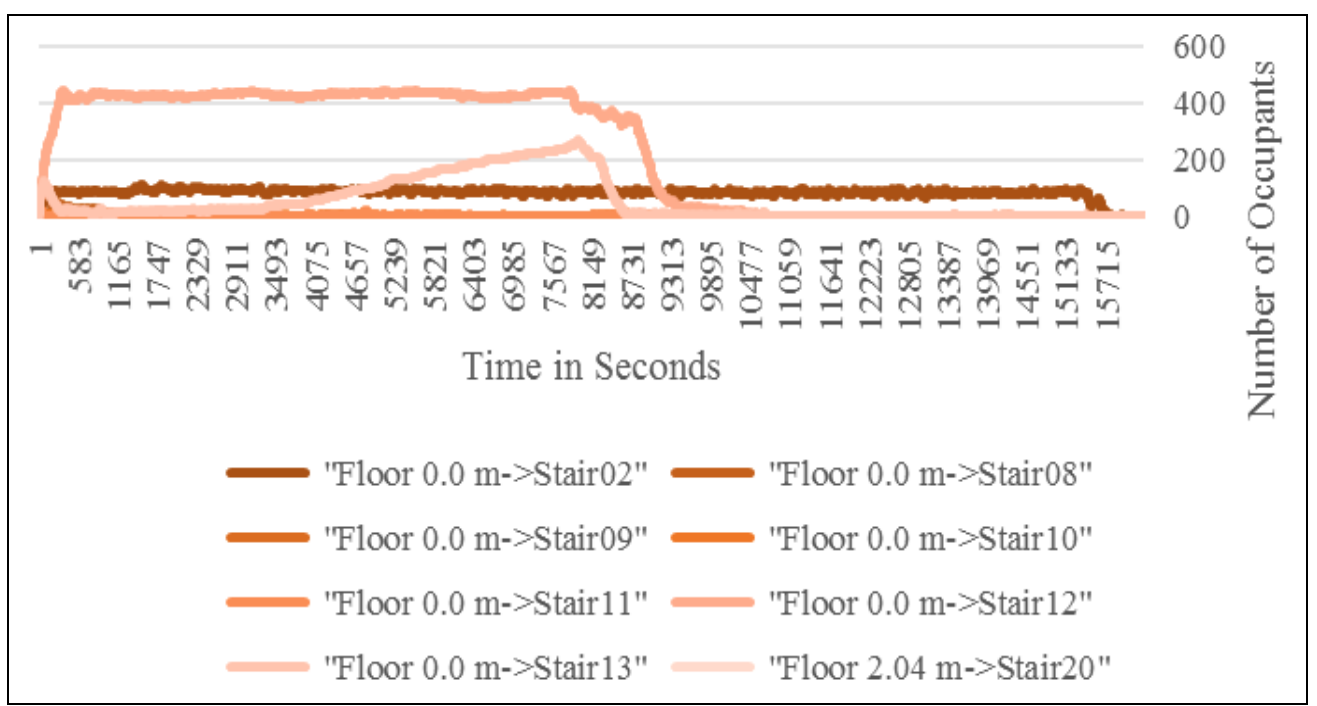

Figure 3: Pedestrian flows at main staircases during evacuation (number of people).

According to the flow rate of gates (Figs. 4 and 5), Gate 3 has the largest pedestrian flow (Fig. 4). Pedestrians pass through these gates almost all the time, and the flow rate is 0.5 pers/s on average. As shown in Fig. 5, the pedestrian flows at Gate 1 are large at 1.2 E04 s. At $1.0 \mathrm{E} 04 \mathrm{~s}$, the flow rate exceeds 1.5 pers/s. In 0 to $5000 \mathrm{~s}$, the pedestrian flows at Gate 2 are large, with the flow rate of approximately 4 pers/s. The pedestrian flows at Gate 6 are divided into two periods of time. In 0 to $1900 \mathrm{~s}$, a large number of pedestrians pass through it with the flow rate of 0.48 pers/s, while in 1900 to $10000 \mathrm{~s}$, almost no one passed through the gate. In 1000 to $13000 \mathrm{~s}$, the pedestrian flows reach a small peak with the maximum flow rate of 1 pers/s. After that, it reduces to 0.2 pers/s. Many pedestrians pass through Gate 5, Gate 4, and Gate 6 in the early stage, but almost no pedestrian in the latter period. The flow rate of Gate 7 reaches 2.3 pers/s in the early stage. Later, only a few pedestrians pass through Gate 7 .

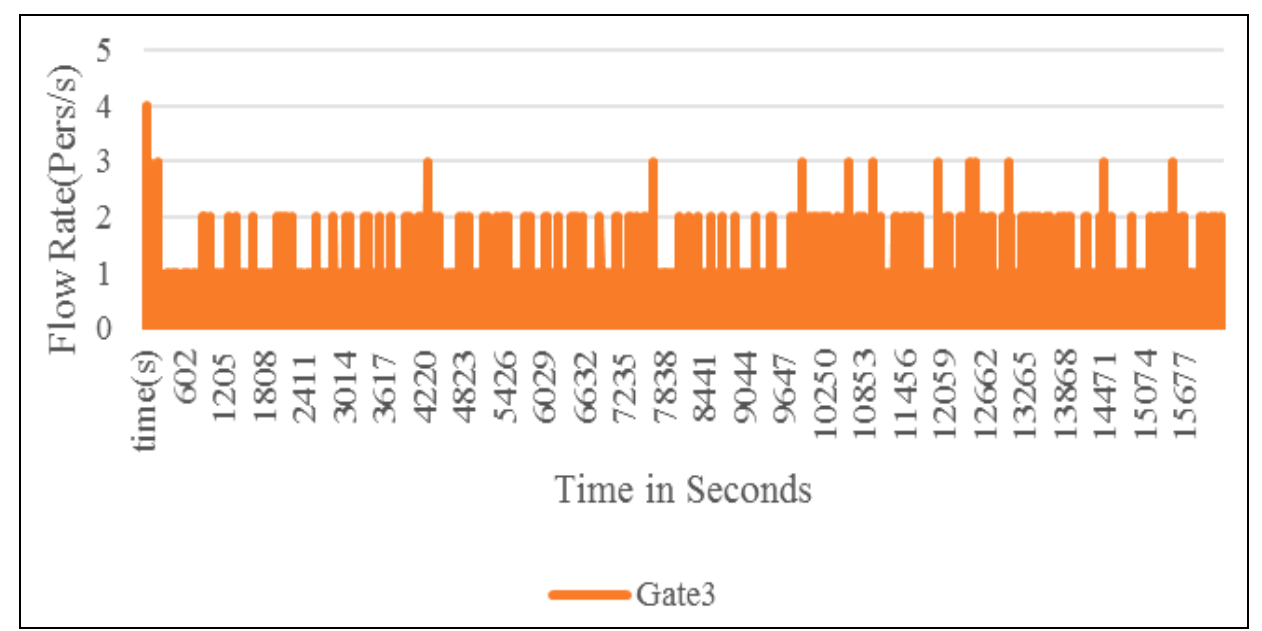

Figure 4: Flow rates for Gate 3 in evacuation (pers/s). 


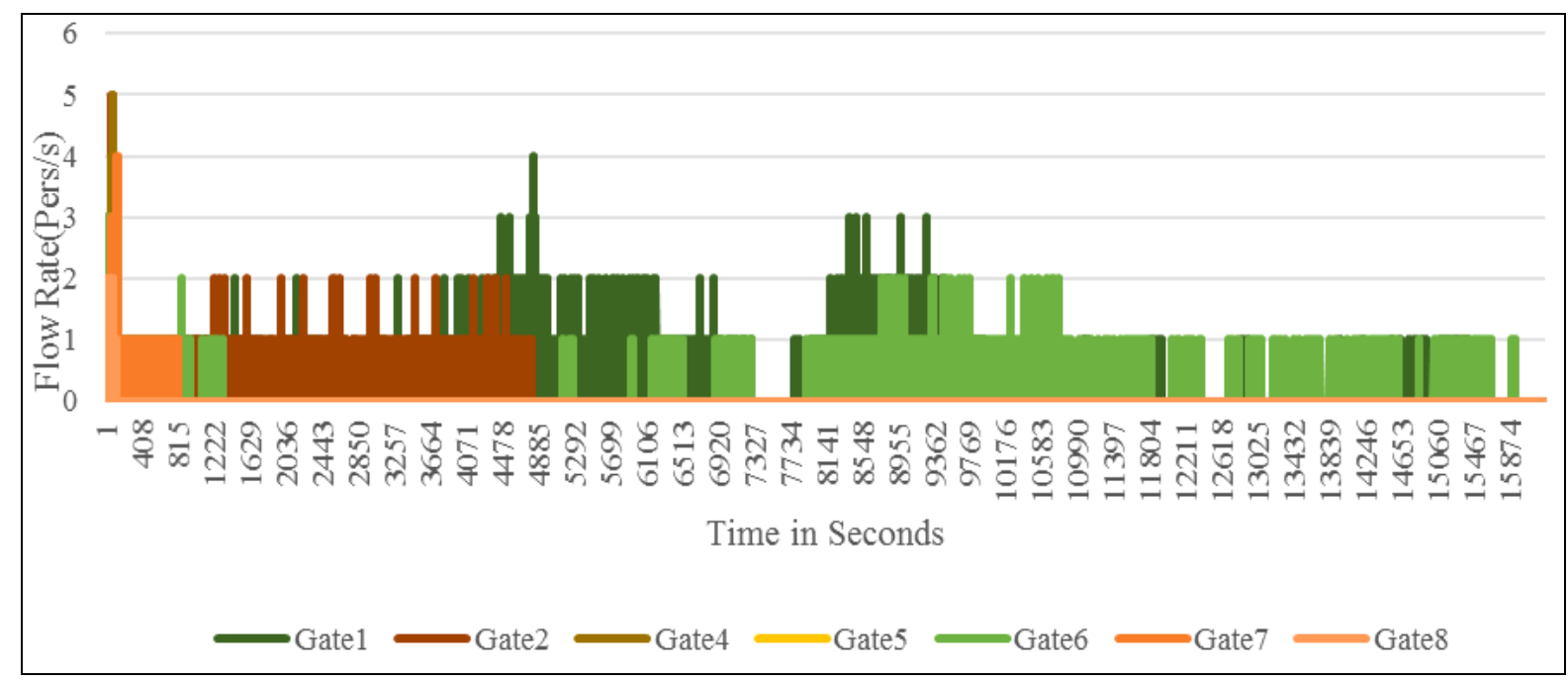

Figure 5: Flow rates for other Gates in evacuation (pers/s).

\subsection{Summary of simulation results}

The 3D simulation results show that the number of evacuees is 233 at $45.8 \mathrm{~s}$ of simulation. Most pedestrians on the first floor are going to the viewing platform layer, and the most pedestrians in the viewing platform layer are going out from the exit. Fig. 6 illustrates that the pedestrians gradually gather, and the crowd density at the place close to the narrow passage of the staircase is high, thereby forming a staircase-centred layout of pedestrians that scatter on different floors. In Fig. 6, the crowd density is higher than 2.79 pedestrians per $\mathrm{m}^{2}$ at Staircases 3 and 4 on the second floor. At Staircase 5, especially the place close to Staircases 3 and 4 , the crowd density is higher than 4.28 pedestrians per $\mathrm{m}^{2}$. In the narrow passage through the east of Chen Yi Square, the crowd density is also high with an average of 3 pedestrians per $\mathrm{m}^{2}$. A medium density area is formed near the high-density area, indicating the transitivity of density aggregation.

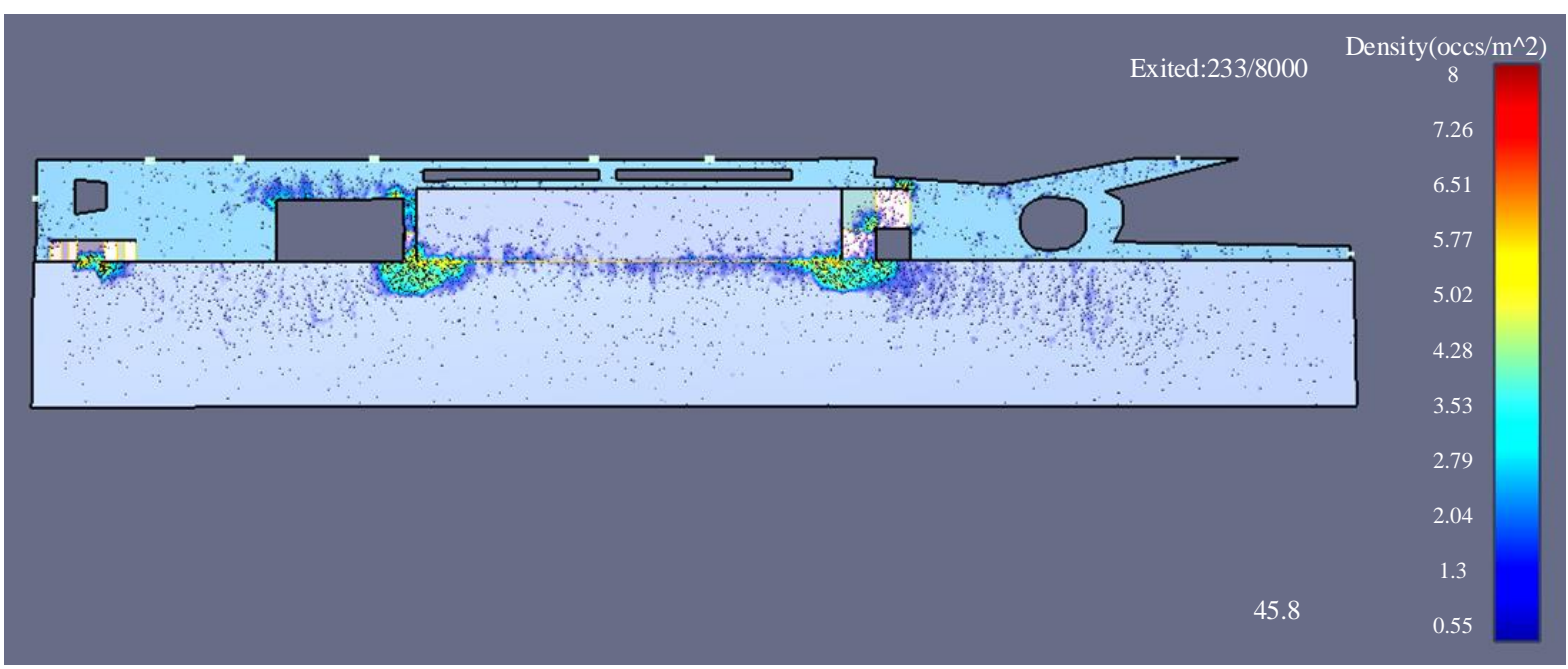

Figure 6: Crowd density at $45.8 \mathrm{~s}$ of evacuation.

A total of 1178 pedestrians have evacuated at $844.8 \mathrm{~s}$ of simulation (Fig. 7). The crowd mainly gathers at Staircases 1, 2, 3, and 4. A fan-shaped and staircase-centred congestion area is formed at Staircases 3 and 4 on the second floor. Pedestrians go up and down the stairs, resulting in large flow hedging, which leads to slow walking speed and crowd evacuation speed. In Fig. 7, the crowd density reaches over 5 pedestrians per square meter at Staircases 1, 
2, 3, and 4. Particularly, a semi-circular waiting area is formed near Staircases 3 and 4 due to congestion.

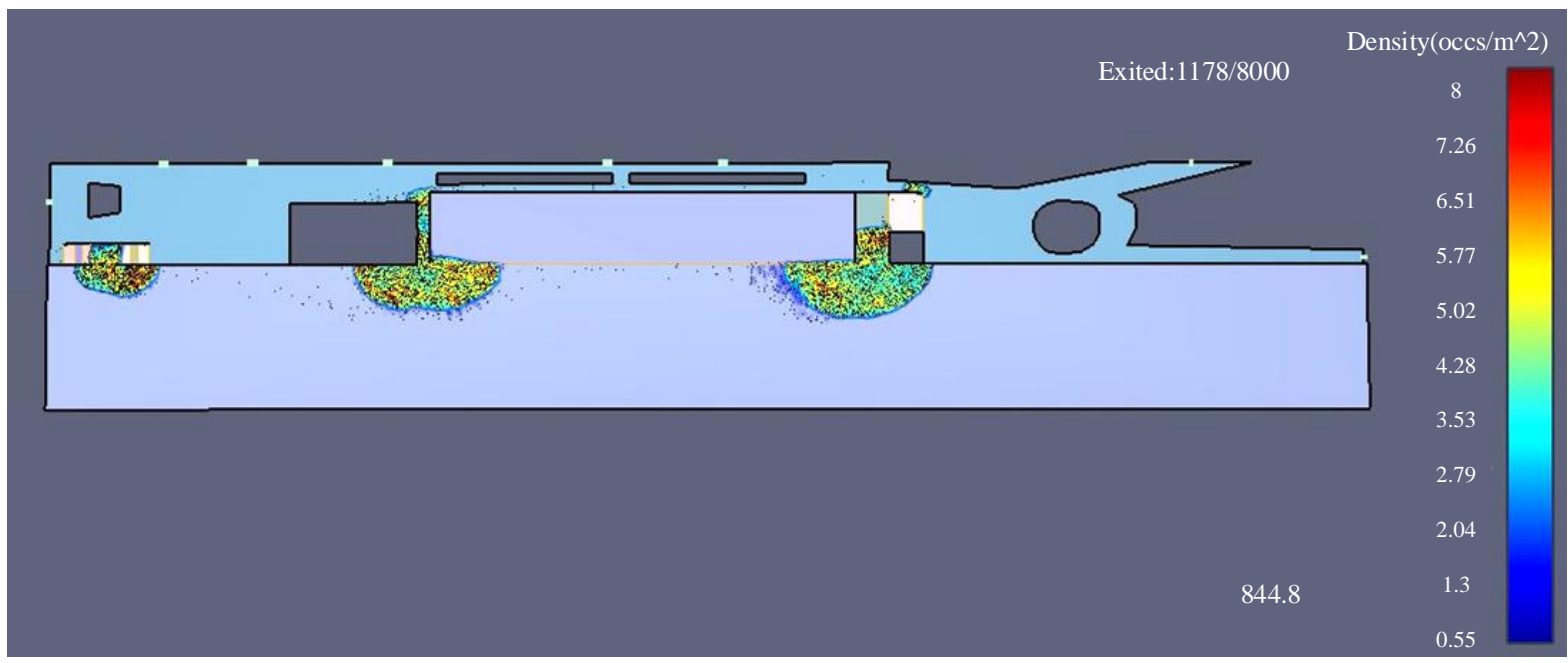

Figure 7: Crowd density at $844.8 \mathrm{~s}$ of evacuation.

The analysis of 3D results suggests that pedestrians are inclined to walk from both sides, thereby showing the typical walking-along-the-sides effect. Fig. 8 shows that pedestrians on both sides form a hedge in the area that connects Staircase 3 in the east of Chen Yi Square. Pedestrians can evacuate rapidly by walking through the gap. Pedestrians in the yellow circle in the figure are walking out of the crow from the side. According to the simulation dynamics, pedestrians are inclined to walk rapidly from gaps on both sides in the state of hedge stalemate. In other words, they incline to keep to the edge of the retrograde and transcendental situations.

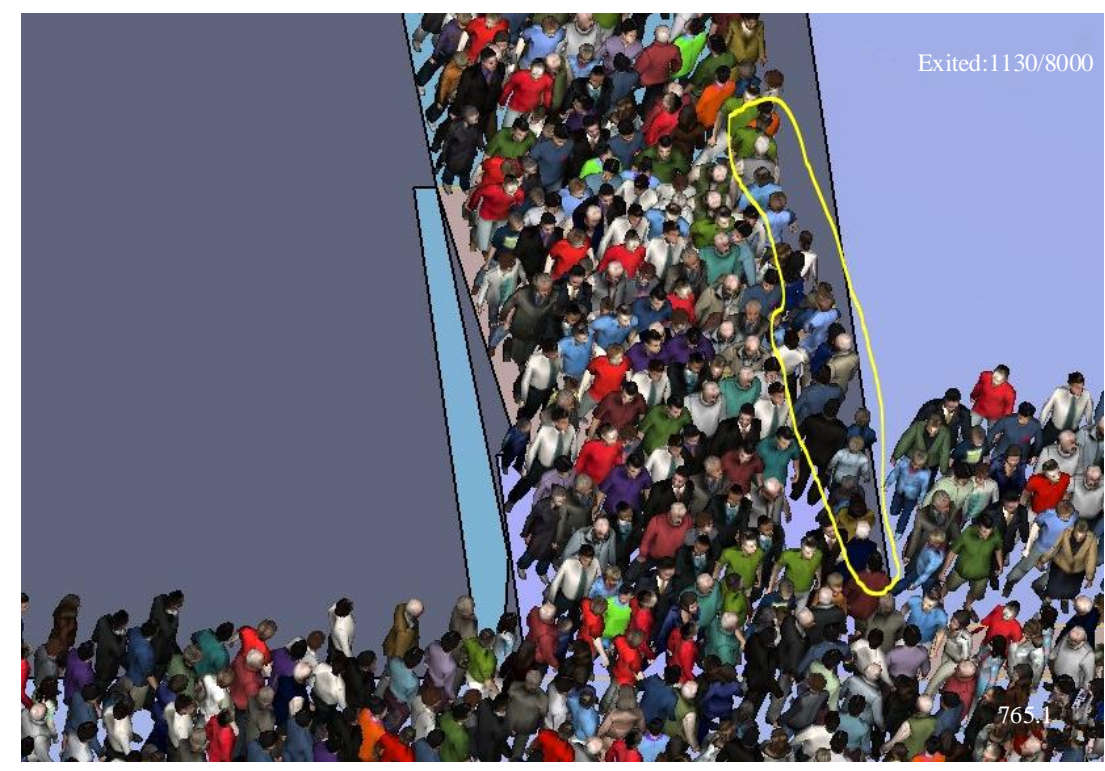

Figure 8: Walking-along-the-sides effect in the process of evacuation.

During evacuation, pedestrians queuing for the end of the line are most likely to change path and select other rapid ways, leading to the imitation effect. Imitation effect means the pedestrians constantly learn and imitate the behaviours of other pedestrians and follow them to move forward. Pedestrians at the end of the queue often have strong imitation effect because they change their path at lower costs. In Fig. 9, pedestrians at the end of the queue at 
the entrance of Staircase 3 on the second floor find the crowd density at Staircases 1 and 2 is lower through observation and learning. Thus, they change their walking paths and run to the entrance of Staircases 1 and 2.

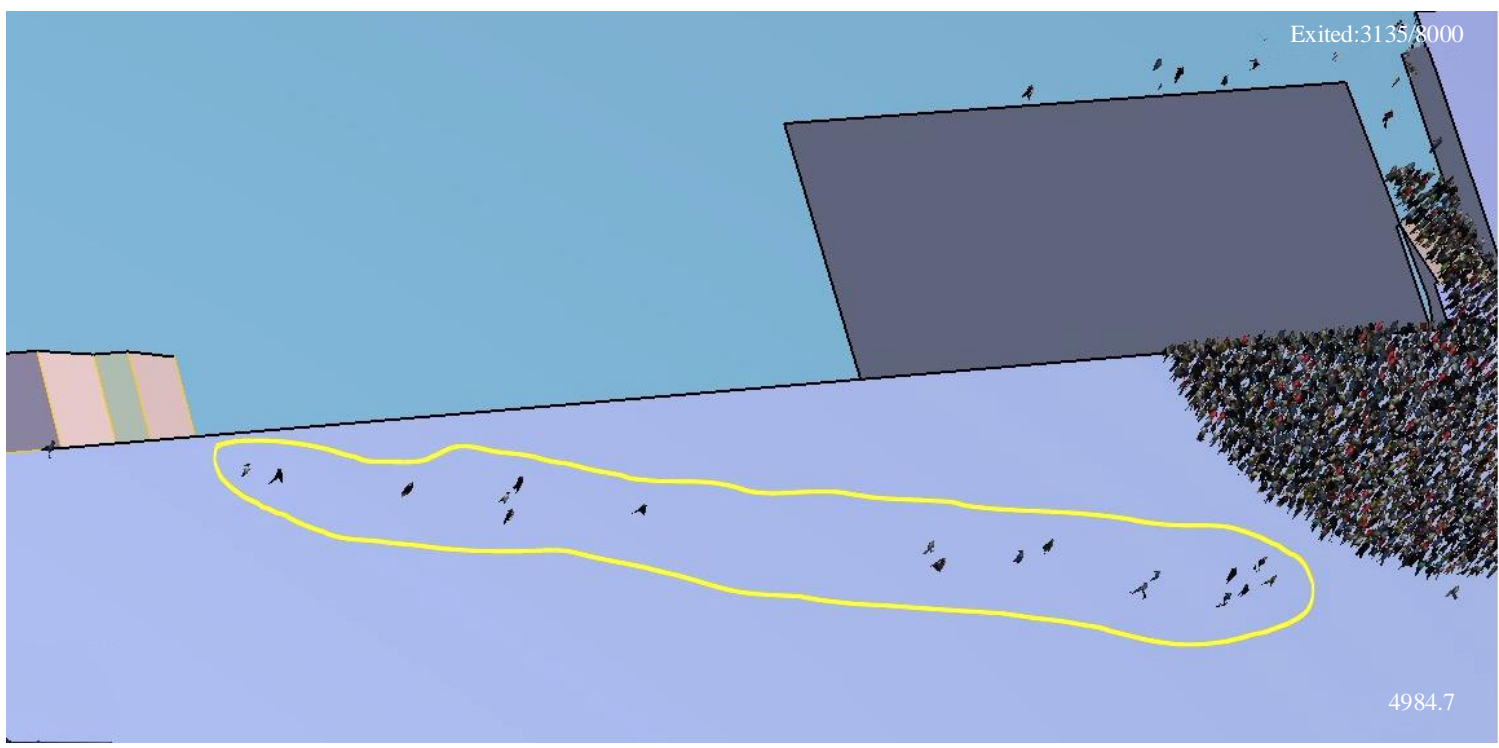

Figure 9: Imitation effect in the process of evacuation.

Pedestrians have the imitation effect and constantly adjust their directions and strategies through imitation in the process of evacuation. Non-optimal effect is evident due to incomplete information. That is, the selected path is not optimal, neither the shortest route nor the most time-saving route. As a result, some pedestrians continue to queue up in the line with slow pedestrian flow, and rare follow the line with sparse pedestrians. For example, in Fig. 10, high pedestrian flow is observed at Staircase 2, but almost no people at Staircases 1 and 3.

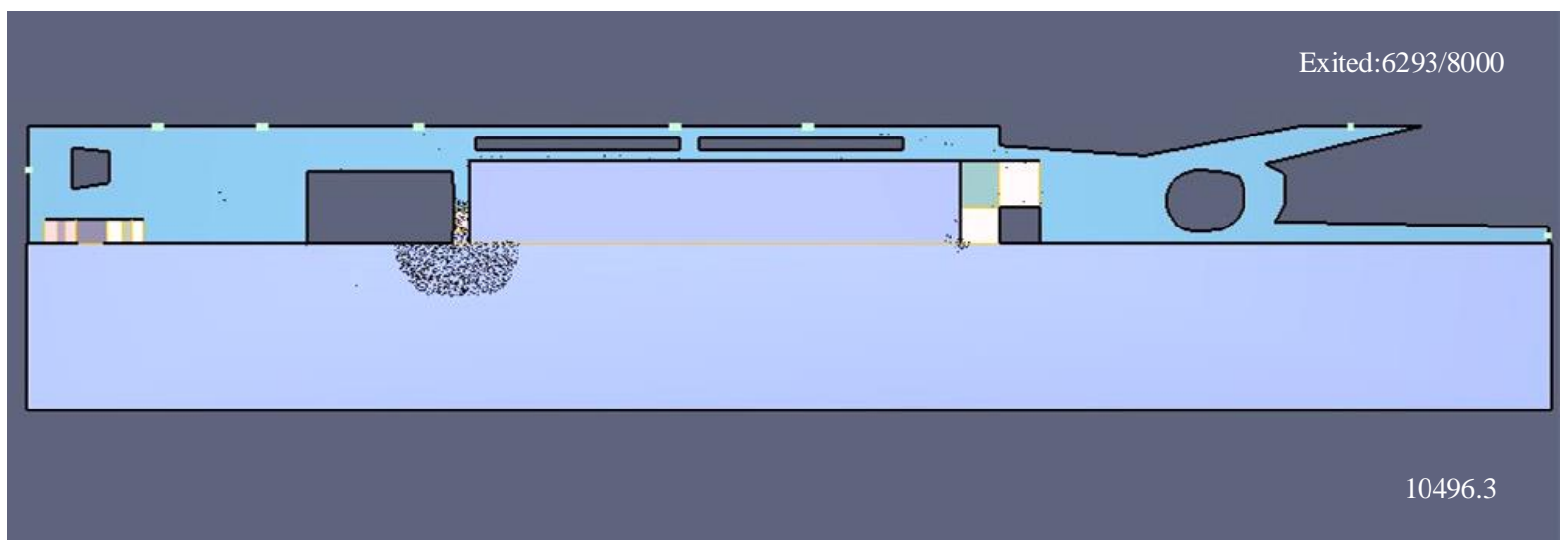

Figure 10: Non-optimal effect in the process of evacuation.

The walking-along-the-sides effect in crowd evacuation, which has not been fully investigated in the previous simulation research [26], could be obtained from simulation. The walking-along-the-sides effect often occurs in dense flow of people. When retrograde behaviour appears, pedestrians are likely to select the sides of the road to evacuate, which is consistent with actual life. On the one hand, guiding pedestrians to evacuate orderly by broadcasting is feasible due to the effect of walking along the sides. On the other hand, safe evacuation zones can be set up on both sides of the crowded area, so that rescuers can arrive at the first time when crowd stampede happens for pedestrians' choosing of walking along the sides. 
The simulation results show that the time of pedestrian evacuation is influenced by pedestrians' physical characteristics, positions, and movement trajectories. Their choices of stairs and gates have limited rationality, which is consistent with the findings of existing research. In real life, pedestrians' evacuation time varies with people and is affected by their physical characteristics, positions, and movement trajectories. Therefore, in daily evacuation management, appropriate guidance strategies according to different characteristics of pedestrians should be considered. At the same time, due to the limited rationality of pedestrians in selecting the evacuation strategy, active evacuation management in crowd places needs to be adopted.

The non-optimal effect in evacuation also occurs frequently in real life, which is manifested in the congestion and queuing of pedestrians in a place due to information asymmetry, especially in staircases, entrances, and exits, while other areas with the same function have lower crowd density or even no people. In real life, video surveillance equipment and public broadcasting system must be installed in crowded places. The management departments should guide the pedestrian flows by using the public broadcasting system based on video surveillance to improve the efficiency of evacuation in time.

\section{CONCLUSION}

To study the evacuation behaviours and the law of crowd evacuation in outdoor public places, the scenarios of 2014 Shanghai Stampede have been constructed to simulate the scenario of crowd evacuation by using Pathfinder based on the consideration of pedestrians' physiological characteristics, movement trajectories, and destinations. The following conclusions are drawn:

(1) The simulation of pedestrians' going up and down shows that evacuation time varies greatly with their locations, trajectories, and physiological characteristics. Evacuation trajectories of different pedestrians vary according to their destinations and evacuation process.

(2) Considering the actual situation of the site and the characteristics of pedestrians, the crowd evacuation effects are simulated and analysed by using Pathfinder, with results presented in 3D form. At the same time, three effects in crowd evacuation, namely, walkingalong-the-sides effect, imitation effect, and non-optimal effect, are found and demonstrated through simulation.

(3) The crowd evacuation effects in public places are simulated and analysed by using Pathfinder. Results show that pedestrians will choose the nearest exit to evacuate in the process of evacuation, without considering other factors. The utilization rates of different gates also have a peak value, which are closely related to pedestrians' path choices, movement speeds, and other factors.

In conclusion, analysing crowd evacuation effects in outdoor public places by constructing a simulation model with Pathfinder can integrate physiological characteristic parameters of individuals and their movement trajectories, taking the complexity of individuals to explore the whole evacuation effects, and further provide theoretical basis of path planning and evacuation guidance in emergency situations. Simulation results show that attentions should be paid to pedestrians' personalized characteristics, especially to the management and control of the sides, staircases, entrances, and exits in narrow areas in evacuation. At the same time, the non-optimal effect of crowd evacuation should be controlled by combining video surveillance system to guide pedestrians to evacuate from free staircases and gates, thereby optimizing evacuation path and saving evacuation time.

However, due to the irregular terrain, complex geographical environment, and other factors in actual measurement, random errors are observed between the measured data and the 
actual situation. Therefore, certain differences from the actual situation can exist when the measurement data is applied to Pathfinder. In addition, to simplify the simulation process, the continuous entry of pedestrians into the site is not considered in the study when the crowd evacuation effects are investigated. Future research can take that into account.

\section{ACKNOWLEDGEMENT}

This work was supported by National Social Science Foundation of China (Project No. 15AGL021).

\section{REFERENCES}

[1] Illiyas, F. T.; Mani, S. K.; Pradeepkumar, A. P.; Mohan, K. (2013). Human stampedes during religious festivals: a comparative review of mass gathering emergencies in India, International Journal of Disaster Risk Reduction, Vol. 5, 10-18, doi:10.1016/j.ijdrr.2013.09.003

[2] Shiwakoti, N.; Tay, R.; Stasinopoulos, P.; Woolley, P. J. (2017). Likely behaviours of passengers under emergency evacuation in train station, Safety Science, Vol. 91, 40-48, doi:10.1016/j.ssci.2016.07.017

[3] Twarogowska, M.; Goatin, P.; Duvigneau, R. (2014). Macroscopic modeling and simulations of room evacuation, Applied Mathematical Modal, Vol. 38, No. 24, 5781-5795, doi:10.1016/ j.apm.2014.03.027

[4] Di Francesco, M.; Markowich, P. A.; Pietschmann, J.-F.; Wolfram, M.-T. (2011). On the Hughes' model for pedestrian flow: the one-dimensional case, Journal of Differential Equations, Vol. 250, No. 3, 1334-1362, doi:10.1016/j.jde.2010.10.015

[5] Zsifkovits, M.; Pham, T. S. (2017). Modelling and parameterizing pedestrian behaviour in public places: a review, International Journal of Simulation Modelling, Vol. 16, No. 4, 630-643, doi:10.2507/IJSIMM16(4)6.399

[6] Guo, X.; Chen, J.; Zheng, Y.; Wei, J. (2012). A heterogeneous lattice gas model for simulating pedestrian evacuation, Physica A: Statistical Mechanics and its Applications, Vol. 391, No. 3, 582-592, doi:10.1016/j.physa.2007.10.001

[7] Hughes, R. L. (2002). A continuum theory for the flow of pedestrians, Transportation Research Part B: Methodological, Vol. 36, No. 6, 507-535, doi:10.1016/S0191-2615(01)00015-7

[8] Kalogeiton, V. S.; Papadopoulos, D. P.; Georgilas, I. P.; Sirakoulis, G. C.; Adamatzky, A. I. (2015). Cellular automaton model of crowd evacuation inspired by slime mould, International Journal of General Systems, Vol. 44, No. 3, 354-391, doi:10.1080/03081079.2014.997527

[9] Zeng, W.; Chen, P.; Nakamura, H.; Iryo-Asano, M. (2014). Application of social force model to pedestrian behaviour analysis at signalized crosswalk, Transportation Research Part C: Emerging Technologies, Vol. 40, 143-159, doi:10.1016/j.trc.2014.01.007

[10] Haghani, M.; Sarvi, M. (2018). Crowd behaviour and motion: empirical methods, Transportation Research Part B: Methodological, Vol. 107, 253-294, doi:10.1016/j.trb.2017.06.017

[11] Henderson, L. F. (1971). The statistics of crowd fluids, Nature, Vol. 229, No. 5284, 381-384, doi: $10.1038 / 229381 \mathrm{a} 0$

[12] Amadori, D.; Di Francesco, M. (2012). The one-dimensional Hughes model for pedestrian flow: Riemann-type solutions, Acta Mathematica Scientia, Vol. 32, No. 1, 259-280, doi:10.1016/ $\underline{\mathrm{S} 0252-9602(12) 60016-2}$

[13] Li, D.; Han, B. (2015). Behavioural effect on pedestrian evacuation simulation using cellular automata, Safety Science, Vol. 80, 41-55, doi:10.1016/j.ssci.2015.07.003

[14] Helbing, D.; Molnar, P. (1995). Social force model for pedestrian dynamics, Physical Review E, Vol. 51, No. 5, 4282-4286, doi:10.1103/PhysRevE.51.4282

[15] Gao, Y.; Chen, T.; Luh, P. B.; Zhang, H. (2016). Modified social force model based on predictive collision avoidance considering degree of competitiveness, Fire Technology, Vol. 53, No. 1, 331351, doi:10.1007/s10694-016-0573-7

[16] Helbing, D.; Isobe, M.; Nagatani, T.; Takimoto, K. (2003). Lattice gas simulation of experimentally studied evacuation dynamics, Physical Review E, Vol. 67, No. 6, Paper 067101, 4 pages, doi: 10.1103/PhysRevE.67.067101 
[17] Guo, X.; Chen, J.; You, S.; Wei, J. (2013). Modeling of pedestrian evacuation under fire emergency based on an extended heterogeneous lattice gas model, Physica A: Statistical Mechanics and its Applications, Vol. 392, No. 9, 1994-2006, doi:10.1016/j.physa.2012.12.033

[18] Huang, K.; Zheng, X.; Cheng, Y.; Yang, Y. (2017). Behaviour-based cellular automaton model for pedestrian dynamics, Applied Mathematics and Computation, Vol. 292, 417-424, doi:10.1016/j.amc.2016.07.002

[19] Helbing, D.; Farkas, I.; Vicsek, T. (2000). Simulating dynamical features of escape panic, Nature, Vol. 407, No. 6803, 487-490, doi:10.1038/35035023

[20] Joo, J.; Kim, N.; Wysk, R. A.; Rothrock, L.; Son, Y.-J.; Oh, Y.-G.; Lee, S. (2013). Agent-based simulation of affordance-based human behaviours in emergency evacuation, Simulation Modelling Practice and Theory, Vol. 32, 99-115, doi:10.1016/j.simpat.2012.12.007

[21] Tan, L.; Hu, M.; Lin, H. (2015). Agent-based simulation of building evacuation: combining human behaviour with predictable spatial accessibility in a fire emergency, Information Sciences, Vol. 295, 53-66, doi:10.1016/j.ins.2014.09.029

[22] Was, J.; Lubas, R. (2014). Towards realistic and effective agent-based models of crowd dynamics, Neurocomputing, Vol. 146, 199-209, doi:10.1016/j.neucom.2014.04.057

[23] Wang, K.; Sheng, W.; Duan, R. (2016). Research on personnel evacuation simulation from exits of university teaching building based on pathfinder, Journal of Safety Science and Technology, Vol. 12, No. 7, 180-186, doi:10.11731/j.issn.1673-193x.2016.07.032

[24] Ding, Y.; Yang, L.; Weng, F.; Fu, Z.; Rao, P. (2015). Investigation of combined stairs elevators evacuation strategies for high rise buildings based on simulation, Simulation Modelling Practice and Theory, Vol. 53, 60-73, doi:10.1016/j.simpat.2015.01.004

[25] Bode, N. W. F.; Codling, E. A. (2013). Human exit route choice in virtual crowd evacuations, Animal Behaviour, Vol. 86, No. 2, 347-358, doi:10.1016/j.anbehav.2013.05.025

[26] Shiwakoti, N.; Sarvi, M. (2013). Understanding pedestrian crowd panic: a review on model organisms approach, Journal of Transport Geography, Vol. 26, 12-17, doi:10.1016/ j.jtrangeo.2012.08.002 